\title{
Spin and orbital Edelstein effects in a two-dimensional electron gas: Theory and application to $\mathrm{SrTiO}_{3}$ interfaces
}

\author{
Annika Johansson $\odot,{ }^{1, *}$ Börge Göbel $\odot,{ }^{1,2}$ Jürgen Henk, ${ }^{1}$ Manuel Bibes $\odot,{ }^{3}$ and Ingrid Mertig ${ }^{1}$ \\ ${ }^{1}$ Institute of Physics, Martin Luther University Halle-Wittenberg, 06099 Halle, Germany \\ ${ }^{2}$ Max Planck Institute of Microstructure Physics, Weinberg 2, 06120 Halle, Germany \\ ${ }^{3}$ Unité Mixte de Physique, CNRS, Thales, Université Paris-Saclay, 91767, Palaiseau, France
}

(Received 26 June 2020; revised 5 February 2021; accepted 5 February 2021; published 24 March 2021)

\begin{abstract}
The Edelstein effect produces a homogeneous magnetization in nonmagnetic materials with broken inversion symmetry which is generated and tuned exclusively electrically. Often the spin Edelstein effect—that is, a spin density in response to an applied electric field-is considered. In this paper we report on the electrically induced magnetization that comprises contributions from the spin and the orbital moments. Our theory for these spin and orbital Edelstein effects is applied to the topologically nontrivial two-dimensional electron gas at $\mathrm{SrTiO}_{3}$ interfaces. In this particular system the orbital Edelstein effect exceeds the spin Edelstein effect by more than one order of magnitude. This finding is explained mainly by orbital moments of different magnitude in the Rashba-like split band pairs, while the spin moments are of almost equal magnitude.
\end{abstract}

DOI: 10.1103/PhysRevResearch.3.013275

\section{INTRODUCTION}

One focus of the field of spintronics is to identify methods which allow us to generate and manipulate spin-polarized electric currents efficiently, with the goal to realize powerful and nonvolatile electronic devices with reduced energy consumption [1]. The initially proposed injection of spinpolarized currents from ferromagnets into semiconductors suffers from unavoidable inefficiency [1,2]. To circumvent this shortcoming, spin-orbitronics aims at generating directly spin-polarized currents in pristine nonmagnetic materials [3-5].

The perhaps most-investigated phenomenon among the vast variety of spin-orbitronic effects is the spin Hall effect: a longitudinal charge current is accompanied by a transversal pure spin current or a spin voltage [6-11]. Phenomenologically similar but of different physical origin is the Edelstein effect, also known as inverse spin-galvanic effect or currentinduced spin polarization [12-14]: in a pristine nonmagnetic system with broken inversion symmetry an applied electric field produces due to spin-orbit coupling a homogeneous spin density perpendicular to the field. A sizable number of systems have been identified which provide efficient chargespin interconversion via the direct or the inverse Edelstein effect [15]: Rashba systems [16-19], semiconductors [20-22], Rashba and Dresselhaus systems with an additional superlattice potential [23,24], topological insulators [25-33],

\footnotetext{
*annika.johansson@physik.uni-halle.de

Published by the American Physical Society under the terms of the Creative Commons Attribution 4.0 International license. Further distribution of this work must maintain attribution to the author(s) and the published article's title, journal citation, and DOI.
}

Weyl semimetals [34], and oxide interfaces featuring twodimensional electron gases (2DEGs) [35-38].

In addition to their spin moment-leading to the spin Hall and the spin Edelstein effect (SEE) - electrons may also carry an orbital moment. Here, two contributions have to be distinguished: on the one hand the electrons' orbital motion and on the other hand the self-rotation of their wave packets [39]. These orbital moments can give rise to the orbital equivalents of the spin Hall and spin Edelstein effects, namely the orbital Hall [40-45] and the orbital Edelstein effect (OEE), the latter producing a current-induced orbital magnetization (Fig. 1).

Although the OEE has been predicted decades ago [46], it is often ignored when Edelstein effects are discussed. Only recently, the OEE resulting from the wave-packet self-rotation has been anticipated for helical and chiral crystals as well as for Rashba systems [47-49]. Furthermore, the SEE and OEE caused by the electrons' orbital motion have been discussed for noncentrosymmetric antiferromagnets [50]. Both SEE and OEE in-plane responses perpendicular to the applied electric field $\mathbf{E}$ were found to be staggered, resulting in a zero net in-plane magnetization perpendicular to E. However, nonzero field-induced magnetization components were predicted to exist parallel to the external field as well as out of plane. Importantly, the calculated orbital contribution to the Edelstein effect is larger than the spin contribution by at least one order of magnitude [50].

The above findings call for a theoretical investigation of the $\mathrm{SEE}$ and $\mathrm{OEE}$ in the 2DEG at $\mathrm{SrTiO}_{3}$ (STO) interfaces, which do not show magnetic order in equilibrium. This particular system lends itself for such a study because of its promising properties concerning spintronics: In particular, the interface between $\mathrm{AlO}_{x}(\mathrm{AO})$ and $\mathrm{SrTiO}_{3}(\mathrm{STO})$ provides a sizable (inverse) Edelstein effect in both theory and experiment, mainly because of its large spin-orbit coupling, topological properties 
(a)

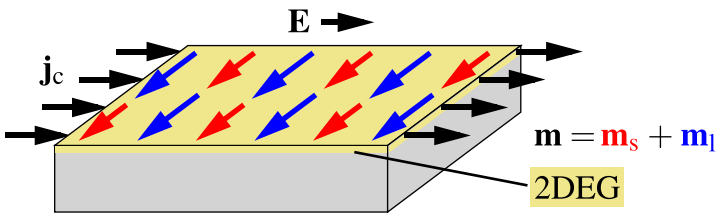

(b)

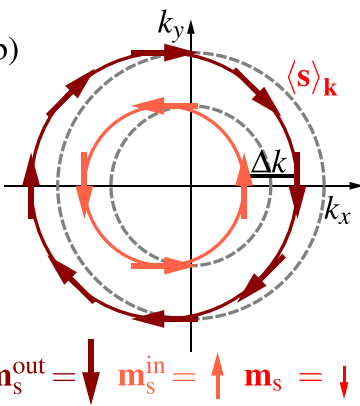

(c)

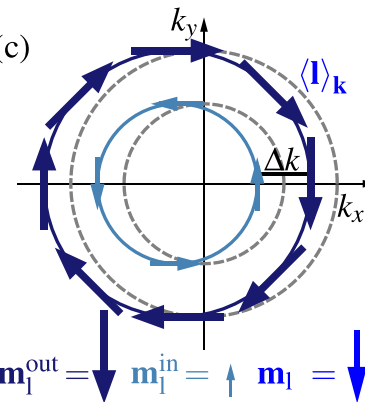

FIG. 1. Spin and orbital Edelstein effects illustrated for a Rashba-split 2DEG with spin- and orbital-momentum locking. An external electric field $\mathbf{E}$ induces a longitudinal charge current $\mathbf{j}_{\mathrm{c}}$ as well as a homogeneous magnetization, usually perpendicular to $\mathbf{E}$. (a) Coexistence of spin $\left(\mathbf{m}_{\mathrm{s}}\right.$, red) and orbital $\left(\mathbf{m}_{\mathrm{l}}\right.$, blue) contributions to the total field-induced magnetic moment $\mathbf{m}$. (b) Spin Edelstein effect. Gray (red) shows equilibrium (nonequilibrium) Fermi contours, arrows show spin expectation values. Due to the nonequilibrium redistribution of states, each Fermi contour provides a finite magnetization ( $\mathbf{m}_{\mathrm{s}}^{\text {out }}$ and $\mathbf{m}_{\mathrm{s}}^{\text {in }}$, respectively). Since these are of opposite sign the total magnetization $\mathbf{m}_{\mathrm{s}}$ is reduced. $\mathbf{m}_{\mathrm{s}}$ is determined by the Rashba splitting $\Delta k$. (c) Orbital Edelstein effect. Similar to panel (b), but here the orbital moments (blue arrows) have different lengths, thereby reducing the compensation and eventually leading to a larger $\mathbf{m}_{1}$.

and a high tunneling barrier preventing the electrons from scattering out of the 2DEG [35,36,38].

Using a semiclassical Boltzmann approach and an effective tight-binding model we calculate the SEE and OEE at STO interfaces as responses to a static electric field. We predict a net OEE originating from the electrons' orbital motion that is more than one order of magnitude larger than its spin companion. Their dependence on the Fermi energy is traced back to band-resolved Edelstein signals. On top of this, we suggest experiments to probe the large orbital contribution to the charge-magnetization interconversion, which is highly favorable for spin-orbitronic applications.

\section{TWO-DIMENSIONAL ELECTRON GAS AT STO INTERFACES}

Although both $\mathrm{SrTiO}_{3}$ and $\mathrm{LaAlO}_{3}$ (LAO) are threedimensional bulk insulators, their two-dimensional (2D) interface features a conducting electron gas [51]. This 2DEG exhibits promising properties, such as high mobility [51], quantum transport [51], tunable carrier density and conductivity [52,53], as well as highly efficient spin-to-charge conversion $[35,36]$. Recently, a 2DEG with similar properties has been found at the (001) surface of STO covered by a thin Al layer [54,55]. This $\mathrm{AlO}_{x} / \mathrm{STO}(\mathrm{AO} / \mathrm{STO}) 2 \mathrm{DEG}$ shows an inverse SEE of enormous magnitude, as is observed in a spin-pumping experiment [38]. Its large signal is mainly

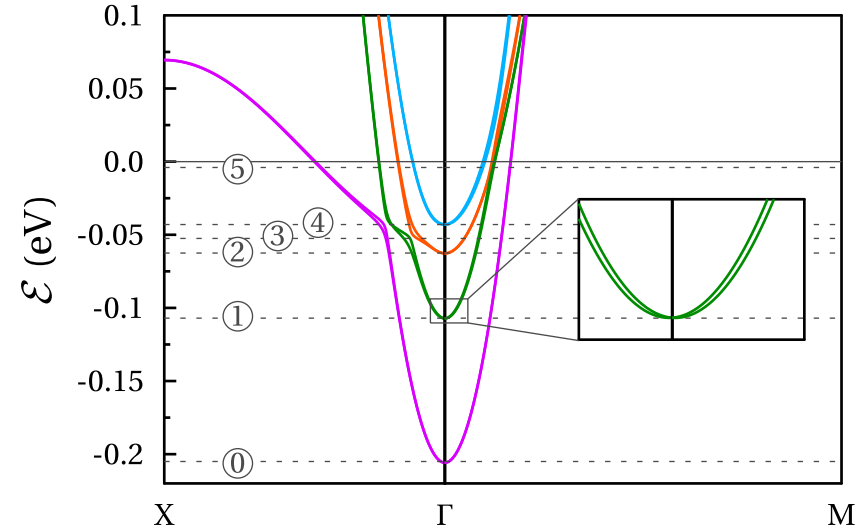

FIG. 2. Band structure of the 2DEG at STO interfaces computed within the tight-binding model. Selected energies are labeled as well as marked by dashed lines: (1) band edge of bands $1+2(-205 \mathrm{meV})$, (1) band edge of bands $3+4(-106 \mathrm{meV})$, (2) band edge of bands $5+6(-63 \mathrm{meV})$, (3) trivial avoided crossing $(-53 \mathrm{meV})$, (4) band edge of bands $7+8(-43 \mathrm{meV})$, and (5) band inversion $(-4 \mathrm{meV})$. The inset shows the Rashba-like splitting at (1).

caused by the interplay of spin-orbit coupling, the topological character of the 2DEG, and the high tunneling resistance of the AO layer. This large (inverse) SEE, mainly originating from $d$ electrons, motivates us to investigate the orbital Edelstein effect in the STO surface 2DEG.

An ideal STO bulk crystal has octahedral symmetry, its Fermi level lies within the fundamental band gap. Even in the presence of spin-orbit coupling the bands are twofold degenerate due to the coexistence of time-reversal and inversion symmetry [56]. Interfaced with LAO or AO, however, the broken inversion symmetry lifts the spin degeneracy [56,57]. Due to the interface constraint, bands originating from the $t_{2 g}$ orbitals are shifted downwards in energy, thereby intersecting the Fermi level. The breaking of inversion symmetry allows for an additional mixing of orbitals (which is forbidden in the bulk) caused by spin-orbit coupling, thereby leading to a Rashba-like splitting of the bands [56,57].

For our investigation we use the effective eight-band tightbinding Hamiltonian proposed in Refs. [38,56-58] to model the $t_{2 g}$ bands relevant for the formation of the 2DEG at the STO surface as well as the LAO-STO and AO-STO interfaces. These systems exhibit a similar band structure; the main difference concerns the charge-carrier density [38,54,58]. For details of the tight-binding model and its parameters [38,58], see Appendix A. The same model has been used in Ref. [38] to calculate the energy-dependent SEE, which agrees well with experimental data for the gate-voltage-dependent inverse SEE. Therefore, the tight-binding model Hamiltonian is expected to reproduce the band structure at the STO interface sufficiently well and lends for a qualitative and demonstrative investigation of the spin and orbital Edelstein effect.

In the energy range around the Fermi level there exist four band pairs: two $d_{x y}$, one $d_{y z}$, and one $d_{z x}$ pair. These band pairs are identified in the band structure (Fig. 2). Spinorbit coupling lifts the twofold spin degeneracy, leading to a Rashba-like splitting near the band edges (inset) and to avoided crossings between the second and third band pairs 
around $-53 \mathrm{meV}$ (label (3)). Vivek et al. predicted that around $-4 \mathrm{meV}$ (5), a band inversion between the second and the third band pairs occurs, accompanied by one-dimensional (1D) spin-polarized helical edge states [58]. This band inversion, characterized by a parity inversion of the eigenstates, is similar to the band inversion of a 2D time-reversal symmetric topological insulator [58,59]. However, the STO surface is not a topological insulator since no fundamental band gap exists near the Fermi level. The band inversion occurs at local band gaps, more precisely, the avoided crossing at (5) in Fig. 2. Due to the topologically nontrivial character of the 2DEG, 1D helical edge states emerge in the energy range around the band inversion. Thus, these edge states are superposed by $2 \mathrm{D}$ bulk states and less exposed than the edge states of $2 \mathrm{D}$ topological insulators [58].

\section{RESULTS: EDELSTEIN EFFECT IN THE STO INTERFACE TWO-DIMENSIONAL ELECTRON GAS}

The current-induced magnetic moment $\mathbf{m}$ per $2 \mathrm{D}$ unit cell is calculated in the linear-response regime,

$$
\frac{A_{0}}{A} \mathbf{m}=\underbrace{\left(\chi^{\mathrm{s}}+\chi^{1}\right)}_{\equiv \chi} \mathbf{E},
$$

where $A_{0}$ is the area of the unit cell, $A$ is the area of the entire system, and $\mathbf{E}$ is the applied electric field. The rank-2 tensors $\chi^{\mathrm{s}}$ and $\chi^{1}$ represent the conversion efficiencies of the SEE (s) and the OEE (l), respectively. Within the semiclassical Boltzmann approach the elements of these tensors read

$$
\begin{aligned}
& \chi_{i j}^{\mathrm{s}}=2 \frac{A_{0} e \mu_{\mathrm{B}}}{A \hbar} \sum_{\mathbf{k}}\langle s\rangle_{\mathbf{k}}^{i} \Lambda_{\mathbf{k}}^{j} \delta\left(\mathcal{E}_{\mathbf{k}}-\mathcal{E}_{\mathrm{F}}\right), \\
& \chi_{i j}^{1}=\frac{A_{0} e \mu_{\mathrm{B}}}{A \hbar} \sum_{\mathbf{k}}\langle l\rangle_{\mathbf{k}}^{i} \Lambda_{\mathbf{k}}^{j} \delta\left(\mathcal{E}_{\mathbf{k}}-\mathcal{E}_{\mathrm{F}}\right),
\end{aligned}
$$

where $i, j=x, y, z, e$ is the elementary charge, $\mu_{\mathrm{B}}$ is the Bohr magneton, $\hbar$ is the reduced Planck constant, and $\hbar \mathbf{k}$ is the crystal momentum. The $\delta$ distributions restrict the band energies $\mathcal{E}_{\mathbf{k}}$ to the Fermi energy $\mathcal{E}_{\mathrm{F}}$. The k-dependent spin expectation value $\langle\mathbf{s}\rangle_{\mathbf{k}}$ and the orbital-momentum expectation value $\langle\mathbf{I}\rangle_{\mathbf{k}}$ are weighted with the mean-free path $\boldsymbol{\Lambda}_{\mathbf{k}}$. The latter is given in constant relaxation-time approximation, $\boldsymbol{\Lambda}_{\mathbf{k}}=\tau_{0} \mathbf{v}_{\mathbf{k}}$ with the relaxation time $\tau_{0}$ and the group velocity $\mathbf{v}_{\mathbf{k}}=\frac{1}{\hbar} \frac{\partial}{\partial \mathbf{k}} \mathcal{E}_{\mathbf{k}}$. The redistribution of the electronic states caused by the external electric field leads to a nonequilibrium magnetization that is attributed either to the spin or to the orbital moments [Figs. 1(b) and 1(c)]. Details of the Boltzmann approach are presented in Appendix B.

The coexistence of time-reversal symmetry and mirror planes perpendicular to the $\langle 100\rangle$ and $\langle 110\rangle$ directions dictates that the spin as well as the orbital moments are oriented completely within the plane. Furthermore, these symmetries allow only nonzero tensor elements $\chi_{x y}=-\chi_{y x}$. In particular, the diagonal elements $\chi_{x x}$ and $\chi_{y y}$ as well as the out-of-plane elements $\chi_{z x}, \chi_{z y}, \chi_{x z}$, and $\chi_{y z}^{\cdot}$, which are predicted in Ref. [50] for noncentrosymmetric antiferromagnets, are forbidden by the mirror symmetries in the STO interfaces.

In isotropic Rashba systems [realized, for example, in the $L$-gap surface state in $\mathrm{Au}(111)$ [60-62]], the spin moments of the Rashba-split states are oriented antiparallel to each other with equal absolute values. Thus, although each individual state would induce a pronounced Edelstein effect, the resulting total SEE from such a pair is strongly reduced due to partial compensation of the oppositely oriented moments, as illustrated in Fig. 1(b).

To discuss the Edelstein effect in STO interfaces, we define the k-dependent quantities $\Delta k, \Delta \Lambda_{\mathbf{k}}, \Sigma_{\mathbf{s}}$, and $\Sigma_{\mathbf{l}}$. These quantities contain properties of two states $\mathbf{k}_{1}$ and $\mathbf{k}_{2}$ which would be degenerate in the absence of spin-orbit coupling. As discussed above, in a Rashba system these states' spin expectation values are oppositely oriented and of equal absolute value, which leads to a pronounced compensation of the Edelstein contributions. An increased Edelstein effect should show up if this compensation is diminished by

(1) a large $\Delta k=\left|k_{1}-k_{2}\right|$, which is due to large spin-orbit interaction and particularly shows up at avoided crossings, accompanied by a large difference of the band-resolved densities of states;

(2) a large difference of mean-free paths $\Delta \Lambda_{\mathbf{k}}=$ $\left|\boldsymbol{\Lambda}_{\mathbf{k}_{1}}-\boldsymbol{\Lambda}_{\mathbf{k}_{2}}\right|$

(3) a large sum $\Sigma_{\mathbf{s}}=\langle\mathbf{s}\rangle_{\mathbf{k}_{1}}+\langle\mathbf{s}\rangle_{\mathbf{k}_{2}}$ (respectively $\Sigma_{\mathbf{l}}=$ $\langle\mathbf{I}\rangle_{\mathbf{k}_{1}}+\langle\mathbf{I}\rangle_{\mathbf{k}_{2}}$ ), meaning that the spins or orbital moments of the Rashba-like-split states are not perfectly aligned antiparallel or differ in absolute value.

The first and second factor affect the SEE and OEE in equal measure, whereas the third may lead to a significantly different charge-magnetic-moment conversion, as sketched in Figs. 1(b) and 1(c). The second point is less relevant for the present study, since we assume a constant relaxation time $\tau_{0}$.

In Rashba systems - and consequently in the present tightbinding model-spin-orbit coupling is necessary to lift spin degeneracy. Splitting the bands of a Kramers pair by $\Delta k$ then produces nonzero spin and orbital moments $\langle\mathbf{s}\rangle_{\mathbf{k}}$ and $\langle\mathbf{l}\rangle_{\mathbf{k}}$. It turns out that hybridization among the set of $t_{2 g}$ orbitals is crucial for the emergence of nonzero orbital moments. A pure $d_{x y}, d_{y z}$, or $d_{z x}$ state would have vanishing expectation values, as is clear by representing the orbital moment $\hat{\mathbf{l}}$ in the $t_{2 g}$ basis (Appendix A).

In Rashba systems with two parabolic bands (free electrons), the spin moments of both bands have the same absolute values; thus, $\Sigma_{\mathbf{s}}$ vanishes. Consequently, the contributions of both bands to the SEE partially compensate. The outer band (with larger $k$ ) dominates and determines the sign of the SEE because of the higher density of states. In the STO interface 2DEG, however, the k-resolved spin and orbital moments exhibit more complex textures than in the free-electron Rashba model, as is evident from Fig. 3. Near the band edges the spin moments exhibit a texture close to that in the free-electron Rashba model-that is equal absolute values for both bands of a pair [Figs. 3(a), 3(c), and 3(e)]; on the contrary, the orbital moments of a band pair are also directed tangentially to their Fermi contour, but the absolute values differ [Figs. 3(b), 3(d), and 3(f)]. This variation is explained by the spin and magnetic quantum numbers. A spin of $s=\frac{1}{2}$ has only two quantum numbers $\left(m_{s}= \pm \frac{1}{2}\right)$, whereas for an orbital moment of $l=2$ ( $d$ orbitals) five $\left(m_{l}=0, \pm 1, \pm 2\right)$ are allowed. This larger variety of quantum numbers is reflected in the expectation values of the orbital moments $\langle\mathbf{I}\rangle_{\mathbf{k}}$, their absolute values can considerably differ among a band pair. 

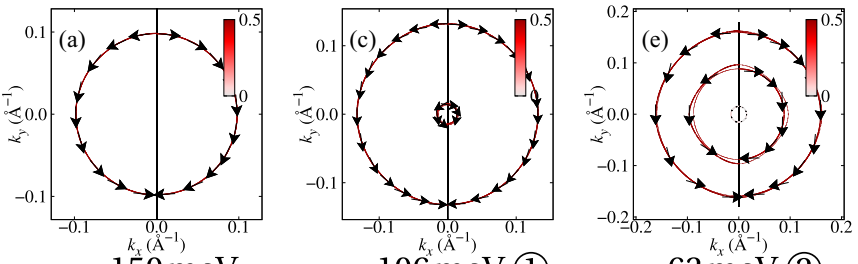

$-150 \mathrm{meV}$
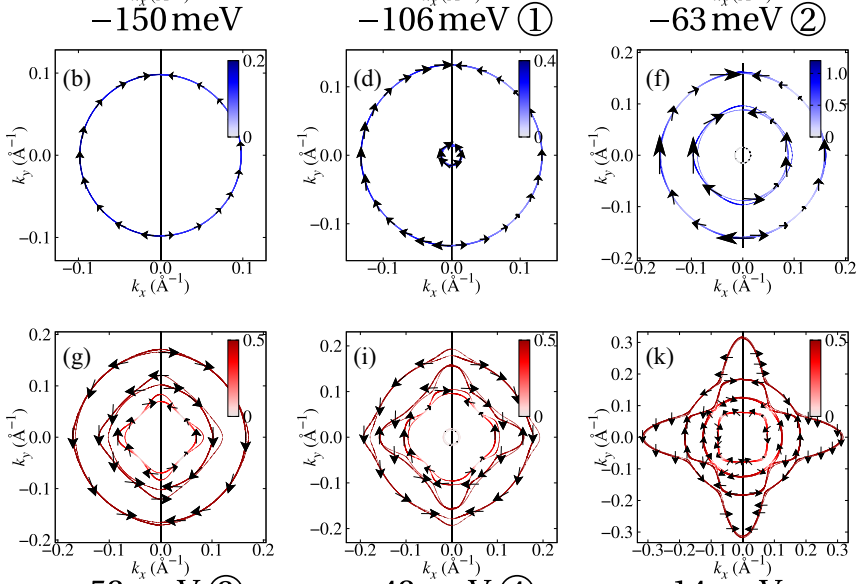

$-53 \mathrm{meV}(3)$
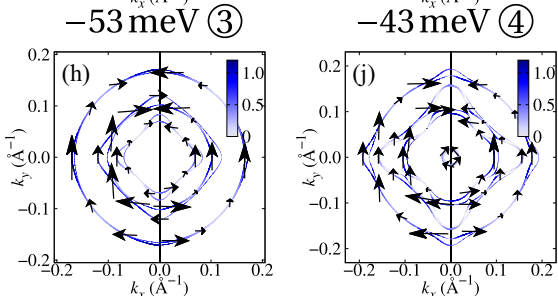

$-14 \mathrm{meV}$

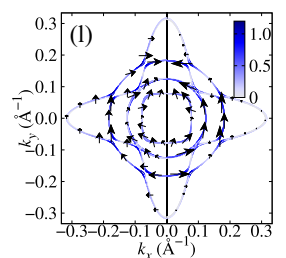

$k_{x}\left(\AA^{-1}\right)$

FIG. 3. Expectation values of spin (red) and orbital (blue) moments (in units of $\hbar$ ) in a STO interface 2DEG at selected iso-energy contours, computed within the tight-binding model. Colors represent the absolute values of the moments. The arrows on the left (right) half of each panel depict the direction of the outer (inner) bands' moments. The encircled labels correspond to the energies indicated in Fig. 2, whereas panels (c)-(f) as well as (i) and (j) show the iso-energy contours slightly above the band edges.

Following up on the above, even a small $\Sigma_{1}$ may produce a sizable OEE that could exceed the SEE [Figs. 1(b) and 1(c)]: the unequal absolute values of $\langle\mathbf{I}\rangle_{\mathbf{k}}$ reduce the compensation of oppositely oriented orbital moments and thereby lead to a more pronounced OEE. As an example consider energies close to the band edge of the lowest band pair. There the OEE surpasses the SEE by a factor of three, although the absolute values of the orbital moments are merely $40 \%$ of the spin moments [Figs. 3(a) and 3(b)] and the mean difference of $\left|\langle\mathbf{I}\rangle_{\mathbf{k}}\right|$ of this Kramers pair amounts to only $3 \%$ of the average spin moment.

This conjecture holds also for energies for which the isoenergy contours deviate strongly from the circular ones of the free-electron Rashba model. As an example consider the contours chosen in Figs. 3(g)-3(1), whose spin and orbital moments exhibit complex textures. The nonzero $\Sigma_{\mathbf{s}}$ [Figs. 3(g), $3(\mathrm{i})$, and $3(\mathrm{k})$ ] yields a sizable SEE. However, $\Sigma_{\mathbf{l}}$ is larger, that is why the OEE is larger as well.

The above examples suggest pronounced signatures of the energy dependencies of the Edelstein efficiencies $\chi_{y x}^{\mathrm{s}}$ and $\chi_{y x}^{1}$ themselves (Fig. 4). The latter are understood in detail by resolving contributions of individual bands.

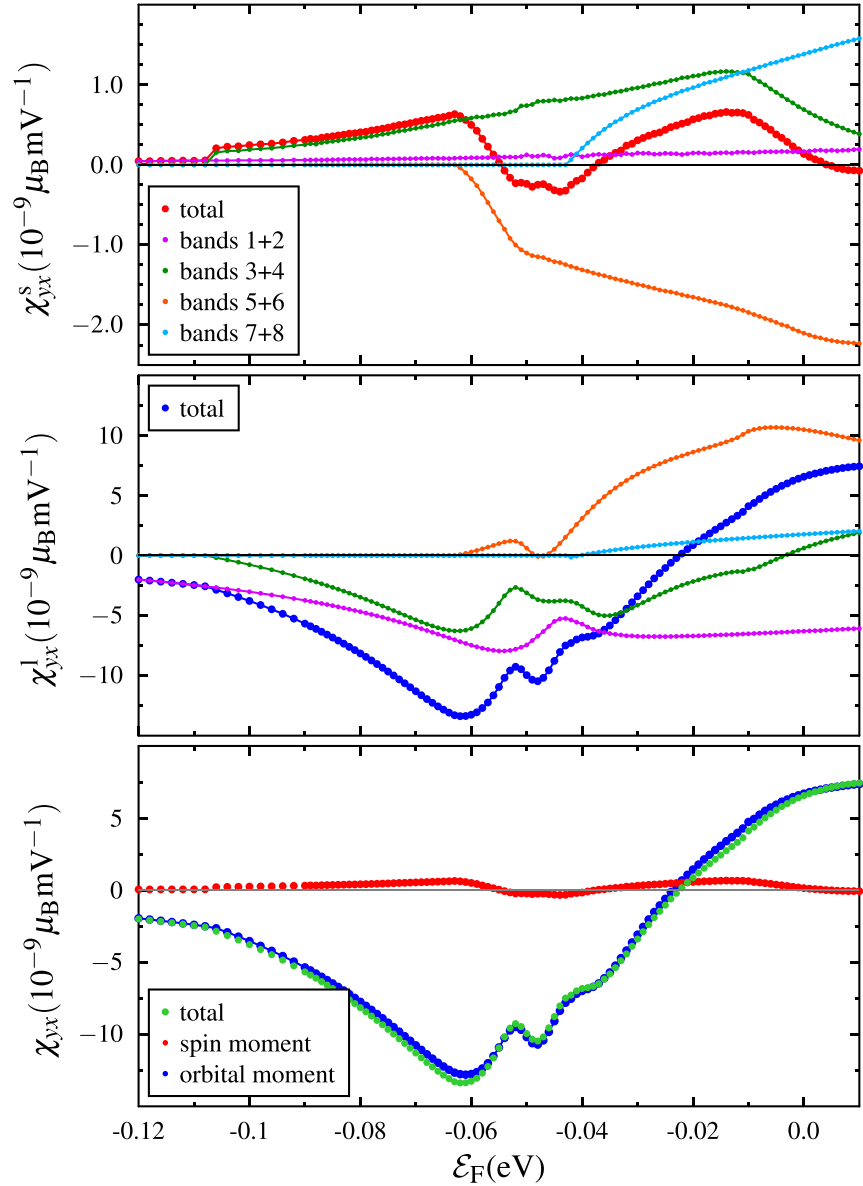

FIG. 4. Efficiency of the spin and orbital Edelstein effects in the STO interface 2DEG calculated within the tight-binding model. The band-resolved spin (a) and orbital (b) contributions as well as the total Edelstein efficiency (c) are shown. Encircled labels indicate the selected energies listed on the right-hand side. The relaxation time is set to $\tau_{0}=1 \mathrm{ps}$.

It suffices to recapitulate briefly the energy dependence of $\chi_{y x}^{\mathrm{s}}$, depicted in Fig. 4(a), since it has been discussed in detail in Ref. [38]. We focus on three effects: density of states, spin texture, and hybridization.

A step-like increase in the total signal at (1) in Fig. 4(a) indicates that the band edge of the second pair increases the number of contributing states. The third Kramers pair has reversed spin chirality with respect to the other pairs [Fig. 3(g)]. This feature explains the opposite sign of $\chi_{y x}^{\mathrm{s}}$ of this pair [orange in Fig. 4(a)] and the extremum of the total signal, clearly showing up at (2). The reversal is compensated by the usual spin chirality of the fourth pair; confer, for example, (4). Uncompensated spin textures produce extrema as well: the maximum near (5) is traced back to the band inversion. However, the strongly increased SEE is not a direct consequence of the band inversion but follows from the peculiar spin texture around the avoided crossing: The spin expectation values of the second and third band pairs strongly deviate from being antiparallel and considerably differ with respect to their moduli, leading to a reduced compensation of the band pairs' contributions to the SEE [enhanced $\Sigma_{\mathbf{s}}$; shown in Fig. 3(k)]. This unconventional spin texture exists in a finite energy range 
around the band inversion. The maximum of the SEE does not occur exactly at the energy of the band inversion, but slightly below $(-14 \mathrm{meV})$. Here, many more states exhibit uncompensated spins than precisely at the energy of the band inversion, which increases the SEE.

Eventually, the signal scales with the splitting $\Delta k$ of a Kramers pair near band edges. $\Delta k$ is enhanced by orbital hybridization which produces the extremum near the avoided crossing at (3).

We now turn to the orbital efficiency $\chi_{y x}^{1}$ whose magnitude is affected by the nonmonotonic behavior of $\Delta k,\left|\left\langle\mathbf{l}_{\mathbf{k}}\right\rangle\right|$, and particularly $\Sigma_{1}$.

As pointed out above, the band-resolved $\left|\langle\mathbf{I}\rangle_{\mathbf{k}}\right|$ of a Kramers pair differ in the free-electron-like regime, in contrast with the $\left|\langle\mathbf{s}\rangle_{\mathbf{k}}\right|$. This reduced compensation $\left(\Sigma_{\mathbf{l}} \neq 0\right)$ leads in combination with $\Delta k$ to a significant but smooth increase of the current-induced magnetization. This signature is exemplified by $\chi_{y x}^{1}$ shown in Fig. 4(b) for bands $1+2$ (() ... (6), bands $3+4$ (1) ... (2)), bands $5+6$ (2) ... (3)), and bands $7+8$ (energies above (4)).

The clear-cut relation of spin chirality and sign of $\chi_{y x}^{\mathrm{s}}$ established above does not hold for $\chi_{y x}^{1}$. Although all band pairs exhibit the same orbital chirality (Fig. 3), the signs of the band-pair-resolved $\chi_{y x}^{1}$ vary because of different distributions of $\langle\mathbf{I}\rangle_{\mathbf{k}}$. For example, the outer states of the pairs $1+2$ and $3+4$ have a larger $\left|\langle\mathbf{I}\rangle_{\mathbf{k}}\right|$ [Figs. 3(b) and 3(d)] as well as a higher density of states than the inner ones; thus, these states determine the sign of the Edelstein signal. Likewise for the other band pairs: here, the "inner" $\left|\langle\mathbf{I}\rangle_{\mathbf{k}}\right|$ are larger than "outer" ones, but despite their lower density of states they set the sign of $\chi_{y x}^{1}$ [Figs. 3(h) and 3(j)].

At elevated energies, the band-resolved efficiencies $\chi_{y x}^{1}$ show a number of extrema. Those at (3), (4), and (7) are related to $\Sigma_{1}$, whereas those at (8), (9), and (10) are associated with $\Delta k$.

The features of the individual bands are carried forward to the total OEE signal; this sum of all orbital contributions exhibits several extrema and a sign change around $-20 \mathrm{meV}$. Abrupt steps or sharp extrema do not occur in $\chi_{y x}^{1}$ because the band-resolved contributions are smooth; this finding is at variance with that for $\chi_{y x}^{\mathrm{s}}$.

Avoided crossings - either topologically trivial (3) or nontrivial (5) — do not lead to pronounced extrema of the orbital signal, although they do so in the spin signal. One consequence of the trivial avoided crossing is to increase $\Delta k$ of the second and third band pair. While this increased splitting implies an enhanced SEE, it does not affect the orbital signal significantly since the latter is partially compensated by the reduction of $\Sigma_{1}$ [Fig. 3(h)]. Furthermore, $\chi_{y x}^{1}$ does not exhibit an extremum at the band inversion because there the emergence of uncompensated orbital moments $\left(\Sigma_{1}\right)$ is less pronounced than that of uncompensated spin moments $\left(\Sigma_{\mathrm{s}}\right)$.

The total Edelstein efficiency $\chi_{y x}$ defined in Eq. (1) is dominated by the orbital contribution which exceeds the spin contribution by one order of magnitude [Fig. 4(c)]. In other words, the fact that $\Sigma_{\mathbf{l}}>\Sigma_{\mathrm{s}}$ for bands of Kramers pair causes $\left|\chi_{y x}^{1}\right|>\left|\chi_{y x}^{\mathrm{s}}\right|$. This implicates that, for any experiment in which the total current-induced magnetization is measured, both spin and orbital moments should be considered as sources of the observed Edelstein effect. This calls for experiments that are able to discriminate both origins.
In general, the SEE and OEE are expected to be not restricted to $\mathrm{SrTiO}_{3}$ interfaces but to exist in a large variety of systems. The most general criterion for both SEE and OEE is broken inversion symmetry. Furthermore, the OEE requires nonzero orbital moments $\langle\mathbf{I}\rangle_{\mathbf{k}}$, a system with only $s$ electrons would not exhibit an OEE but possibly a SEE. A sizable OEE is expected in materials with large spin-orbit interaction, large relaxation times, and high orbital quantum numbers. Both Edelstein effects are enhanced by large spinorbit coupling, which lifts band degeneracies and therefore reduces the compensation of neighboring bands' contributions to the SEE and OEE. Higher orbital quantum numbers lead to a reduced compensation of orbital moments of band pairs because of the larger variety of magnetic quantum numbers. From a practical perspective, systems with minor scattering from the 2DEG into the adjacent materials are favorable, such as the $\mathrm{AlO}_{x}-\mathrm{SrTiO}_{3}$ interface [38].

\section{DETECTION OF THE ORBITAL EDELSTEIN EFFECT}

A vast number of experiments address the charge-spin interconversion via the direct or the inverse Edelstein effect; to name a few: Refs. [20-22,28-31,35,37,38,63-71]. These are usually interpreted within a theoretical framework in which merely the spin contribution to the Edelstein effect is considered. However, as we have demonstrated in the previous section, the orbital contribution cannot be neglected a priori.

In a system with broken inversion symmetry the magnetization induced by the direct Edelstein effect would be of both spin and orbital origin, unless $s$ electrons $(l=0)$ dominate the transport.

To detect the direct orbital Edelstein effect, one may resort to probes able to separate the spin and orbital contribution of induced magnetization such as X-ray magnetic circular dichroism (XMCD) [72]. Running a current in a STO 2DEG channel and measuring the XMCD at the Ti $L_{3,2}$ edge may thus allow for the experimental observation of the OEE in this material. Importantly, however, the finite overlap between $L_{2}$ and $L_{3}$ edges for Ti complicates the quantitative deduction of the spin and orbital moments through the so-called sum rules methods $[73,74]$, implying that this analysis would have to be backed by multiplet calculations. For other systems based on heavier elements with a better separation of $L_{2}$ and $L_{3}$ edges, the application of sum rules should directly yield the spin and orbital contributions.

\section{SYNOPSIS}

Our theoretical investigation of the spin and orbital Edelstein effects in the 2DEG at a STO interface reveals that the contribution from the orbital moments exceeds that of the spin moments by more than one order of magnitude. This finding is explained mainly by larger variations of the band-resolved orbital moments along the Fermi contour as compared with those of the spin moments. From this follows that the orbital contribution proves significant in applications which rely on the direct Edelstein effects. The orbital Edelstein effect could be distinguished from its spin counterpart by XMCD experiments.

Applications featuring an inverse Edelstein effect, e.g., the proposed magnetoelectric spin-orbit device [75], would 
be more efficient by utilizing the inverse OEE, that is, by injecting an orbital current in addition to a spin current.

\section{ACKNOWLEDGMENTS}

This work is supported by CRC/TRR 227 of Deutsche Forschungsgemeinschaft (DFG) and the ERC Advanced Grant No. 833973 "FRESCO." M.B. thanks the Alexander von Humboldt Foundation for supporting his stays at Martin Luther University Halle-Wittenberg.

\section{APPENDIX A: ELECTRONIC-STRUCTURE CALCULATIONS}

The relevant electronic states that form the $2 \mathrm{DEG}$ at the STO interface are derived from four $t_{2 g}$ orbitals [56,57]. It is thus natural to utilize the tight-binding Hamiltonian proposed in Refs. [56-58] and its extension to eight bands proposed in Ref. [38].

The basis set consists of four spin-up orbitals- $\left\{d_{x y \uparrow}^{(1)}, d_{x y \uparrow}^{(2)}, d_{y z \uparrow}, d_{z x \uparrow}\right\}$-and four spin-down orbitals- $\left\{d_{x y \downarrow}^{(1)}, d_{x y \downarrow}^{(2)}, d_{y z \downarrow}, d_{z x \downarrow}\right\}$. The superscripts 1 and 2 for the $d_{x y}$ orbitals account for crystal-field splitting; see Eq. (A5) below. The Hamiltonian matrix

$$
\hat{H}=\left(\begin{array}{ll}
H^{+} & H_{\lambda} \\
H_{\lambda}^{\dagger} & H^{-}
\end{array}\right)
$$

decomposes thus into the blocks

$$
H^{ \pm}=\left(\begin{array}{cccc}
\mathcal{E}_{x y}^{(1)} & 0 & i g_{1} \sin \tilde{k}_{x} & i g_{1} \sin \tilde{k}_{y} \\
0 & \mathcal{E}_{x y}^{(2)} & i g_{2} \sin \tilde{k}_{x} & i g_{2} \sin \tilde{k}_{y} \\
-i g_{1} \sin \tilde{k}_{x} & -i g_{2} \sin \tilde{k}_{x} & \mathcal{E}_{y z} & \pm i \lambda \\
-i g_{1} \sin \tilde{k}_{y} & -i g_{2} \sin \tilde{k}_{y} & \mp i \lambda & \mathcal{E}_{z x}
\end{array}\right)
$$

and

$$
H_{\lambda}=\lambda\left(\begin{array}{cccc}
0 & 0 & 1 & -i \\
0 & 0 & 1 & -i \\
-1 & -1 & 0 & 0 \\
i & i & 0 & 0
\end{array}\right)
$$

with $\tilde{k}_{i}=k_{i} a$ ( $a$ lattice constant). The diagonal terms

$$
\begin{aligned}
\mathcal{E}_{x y}^{(i)} & =2 t\left(2-\cos \tilde{k}_{x}-\cos \tilde{k}_{y}\right)+\mathcal{E}_{0 x y}^{(i)}, \quad i=1,2, \\
\mathcal{E}_{y z} & =2 t\left(1-\cos \tilde{k}_{y}\right)+2 t_{h}\left(1-\cos \tilde{k}_{x}\right)+\mathcal{E}_{0 z}, \\
\mathcal{E}_{z x} & =2 t\left(1-\cos \tilde{k}_{x}\right)+2 t_{h}\left(1-\cos \tilde{k}_{y}\right)+\mathcal{E}_{0 z},
\end{aligned}
$$

reflect the band structure without spin-orbit coupling. $t$ and $t_{h}$ denote the strength of nearest-neighbor hopping of the light and the heavy bands, respectively. Terms proportional to $\lambda$ mimic atomic spin-orbit coupling, while those proportional to $g_{1}$ or $g_{2}$ account for the interatomic and orbital-mixing spin-orbit interaction, which stems from the deformation of the orbitals at the interface $[56,57,76]$. The parameters for the STO interface,

$$
\begin{aligned}
\mathcal{E}_{0 x y}^{(1)} & =-205 \mathrm{meV}, & t & =388 \mathrm{meV}, \\
\mathcal{E}_{0 x y}^{(2)} & =-105 \mathrm{meV}, & t_{h} & =31 \mathrm{meV}, \\
\mathcal{E}_{0 z} & =-54 \mathrm{meV}, & g_{1} & =2 \mathrm{meV}, \\
\lambda & =8.3 \mathrm{meV}, & g_{2} & =5 \mathrm{meV},
\end{aligned}
$$

are taken from Ref. [58]. These were obtained by fitting the band structure to experimental photoemission data and were successfully used in Ref. [38].

The expectation value

$$
\langle\mathbf{s}\rangle_{\mathbf{k}}^{n}=\left\langle\Psi_{\mathbf{k}}^{n}|\hat{\mathbf{s}}| \Psi_{\mathbf{k}}^{n}\right\rangle
$$

of the spin operator $\hat{\mathbf{s}}$ with respect to a Bloch state $\left|\Psi_{\mathbf{k}}^{n}\right\rangle$ (n band index) is expressed in terms of the Pauli matrices,

$$
\hat{s}_{i}=\hat{\sigma}_{i} \otimes \mathbb{1}_{4 \times 4}, \quad i=x, y, z .
$$

The expectation value of the orbital moment $\hat{I}$ reads

$$
\langle\mathbf{l}\rangle_{\mathbf{k}}^{n}=\left\langle\Psi_{\mathbf{k}}^{n}|\hat{\mathbf{l}}| \Psi_{\mathbf{k}}^{n}\right\rangle
$$

with

$$
\hat{l}_{i}=\mathbb{1}_{2 \times 2} \otimes \hat{\lambda}_{i}
$$

and

$$
\begin{aligned}
\hat{\lambda}_{x} & =\left(\begin{array}{cccc}
0 & 0 & 0 & -i \\
0 & 0 & 0 & -i \\
0 & 0 & 0 & 0 \\
i & i & 0 & 0
\end{array}\right), \\
\hat{\lambda}_{y} & =\left(\begin{array}{cccc}
0 & 0 & i & 0 \\
0 & 0 & i & 0 \\
-i & -i & 0 & 0 \\
0 & 0 & 0 & 0
\end{array}\right), \\
\hat{\lambda}_{z} & =\left(\begin{array}{cccc}
0 & 0 & 0 & 0 \\
0 & 0 & 0 & 0 \\
0 & 0 & 0 & i \\
0 & 0 & -i & 0
\end{array}\right) .
\end{aligned}
$$

\section{APPENDIX B: TRANSPORT CALCULATIONS}

The magnetic moment $\mathbf{m}_{\mathrm{s}}$ originating from the spin moment is given by

$$
\mathbf{m}_{\mathrm{s}}=-\frac{g_{s} \mu_{\mathrm{B}}}{\hbar} \sum_{\mathbf{k}} f_{\mathbf{k}}\langle\mathbf{s}\rangle_{\mathbf{k}}
$$

in the semiclassical Boltzmann theory for transport utilized here. Here and in the following, the multi-index $\mathbf{k}$ comprises the crystal momentum as well as the band index, $\mathbf{k} \equiv(\hbar \mathbf{k}, n)$. For the LAO-STO interface, Landé's $g$ factors between 0.8 and 5.2 have been reported [77-80]. Since the main purpose of this paper is to predict the existence of the OEE at STO interfaces and not to calculate exact numbers, and since the experimentally determined values of the $g$ factor at the LAOSTO interface are of the same order of magnitude as the free electron $g$ factor, we chose $g_{s} \approx 2$ here.

Likewise, the orbital moment yields a magnetic moment

$$
\mathbf{m}_{1}=-\frac{g_{l} \mu_{\mathrm{B}}}{\hbar} \sum_{\mathbf{k}} f_{\mathbf{k}}\langle\mathbf{I}\rangle_{\mathbf{k}}
$$

with $g_{l}=1$.

The distribution function $f_{\mathbf{k}}=f_{\mathbf{k}}^{0}+g_{\mathbf{k}}$ is split into an equilibrium part-that is the Fermi-Dirac distribution function 
$f_{\mathbf{k}}^{0}$ - and a nonequilibrium part $g_{\mathbf{k}}$. In nonmagnetic systems, $f_{\mathbf{k}}^{0}$ does not contribute to $\mathbf{m}_{\mathrm{s}}$ and $\mathbf{m}_{\mathrm{l}}$.

The Boltzmann equation for a stationary and spatially homogeneous system reads

$$
\dot{\mathbf{k}} \frac{\partial f_{\mathbf{k}}}{\partial \mathbf{k}}=\left(\frac{\partial f_{\mathbf{k}}}{\partial t}\right)_{\text {scatt }} .
$$

Using the semiclassical equation of motion

$$
\dot{\mathbf{k}}=-\frac{e}{\hbar} \mathbf{E}
$$

expressing the right-hand side in terms of microscopic transition probability rates $P_{\mathbf{k}^{\prime} \leftarrow \mathbf{k}}$,

$$
\left(\frac{\partial f_{\mathbf{k}}}{\partial t}\right)_{\text {scatt }}=\sum_{\mathbf{k}^{\prime}}\left(P_{\mathbf{k} \leftarrow \mathbf{k}^{\prime}} g_{\mathbf{k}^{\prime}}-P_{\mathbf{k}^{\prime} \leftarrow \mathbf{k}} g_{\mathbf{k}}\right),
$$

and with the linear ansatz

$$
g_{\mathbf{k}}=\frac{\partial f_{\mathbf{k}}^{0}}{\partial \mathcal{E}} e \boldsymbol{\Lambda}_{\mathbf{k}} \cdot \mathbf{E},
$$

the Boltzmann equation is linearized and takes the form

$$
\boldsymbol{\Lambda}_{\mathbf{k}}=\tau_{\mathbf{k}}\left(\mathbf{v}_{\mathbf{k}}+\sum_{\mathbf{k}^{\prime}} P_{\mathbf{k} \leftarrow \mathbf{k}^{\prime}} \Lambda_{\mathbf{k}^{\prime}}\right)
$$

for the mean-free path $\boldsymbol{\Lambda}_{\mathbf{k}}$. The momentum relaxation time is given by

$$
\tau_{\mathbf{k}}=\left(\sum_{\mathbf{k}^{\prime}} P_{\mathbf{k}^{\prime} \leftarrow \mathbf{k}}\right)^{-1} .
$$

For the calculations presented in this paper we neglect the so-called scattering-in term $\tau_{\mathbf{k}} \sum_{\mathbf{k}^{\prime}} P_{\mathbf{k} \leftarrow \mathbf{k}^{\prime}} \boldsymbol{\Lambda}_{\mathbf{k}^{\prime}}$ which appears in Eq. (B7). This approximation is adequate because the scattering-in term would modify the numerical results only slightly, and the main purpose of this paper is to predict the existence of the OEE in addition to the SEE as well as to demonstrate that the OEE can be larger than the SEE. With the further assumptions of zero temperature and a constant relaxation time $\tau_{\mathbf{k}}=\tau_{0}$, Eqs. (1) and (2) yield the magnetic moment induced by an external electric field within the linear-response regime. The constant relaxation-time approximation has provided good qualitative agreement with the experimentally observed inverse spin Edelstein effect at the AO-STO interface [38]. In the experimental setup, electrons can scatter out of the 2DEG to neighboring materials. This additional scattering channel reduces inhomogeneities of the relaxation time with respect to $\mathbf{k}$ and the energy; therefore, the constant relaxationtime approach is appropriate. Using a k- and energydependent relaxation time instead of the constant $\tau_{0}$ would further modify the energy dependence of the OEE and SEE.
[1] S. A. Wolf, D. D. Awschalom, R. A. Buhrman, J. M. Daughton, S. von Molnár, M. L. Roukes, A. Y. Chtchelkanova, and D. M. Treger, Spintronics: A spin-based electronics vision for the future, Science 294, 1488 (2001).

[2] G. Schmidt, Concepts for spin injection into semiconductors-a review, J. Phys. D: Appl. Phys. 38, R107 (2005).

[3] A. Manchon, H. C. Koo, J. Nitta, S. M. Frolov, and R. A. Duine, New perspectives for Rashba spin-orbit coupling, Nat. Mater. 14, 871 (2015).

[4] A. Soumyanarayanan, N. Reyren, A. Fert, and C. Panagopoulos, Emergent phenomena induced by spin-orbit coupling at surfaces and interfaces, Nature (London) 539, 509 (2016).

[5] A. Manchon and A. Belabbes, Spin-orbitronics at transition metal interfaces, Adv. At. Mol. Opt. Phys. 68, 1 (2017).

[6] N. F. Mott and H. S. W. Massey, Theory of Atomic Collisions (Oxford University Press, Oxford, 1965).

[7] L. D. Landau and E. M. Lifshitz, Quantum Mechanics: NonRelativistic Theory (Pergamon Press, Oxford, 1965).

[8] M. Dyakonov and V. Perel, Current-induced spin orientation of electrons in semiconductors, Phys. Lett. A 35, 459 (1971).

[9] J. E. Hirsch, Spin Hall Effect, Phys. Rev. Lett. 83, 1834 (1999).

[10] Y. K. Kato, R. C. Myers, A. C. Gossard, and D. D. Awschalom, Observation of the spin Hall effect in semiconductors, Science 306, 1910 (2004).

[11] J. Sinova, S. O. Valenzuela, J. Wunderlich, C. H. Back, and T. Jungwirth, Spin Hall effects, Rev. Mod. Phys. 87, 1213 (2015).

[12] A. G. Aronov and Y. B. Lyanda-Geller, Nuclear electric resonance and orientation of carrier spins by an electric field, JETP Lett. 50, 431 (1989).
[13] V. M. Edelstein, Spin polarization of conduction electrons induced by electric current in two-dimensional asymmetric electron systems, Solid State Commun. 73, 233 (1990).

[14] P. Gambardella and I. M. Miron, Current-induced spin-orbit torques, Philos. Trans. R. Soc., A 369, 3175 (2011).

[15] K. Shen, G. Vignale, and R. Raimondi, Microscopic Theory of the Inverse Edelstein Effect, Phys. Rev. Lett. 112, 096601 (2014).

[16] E. Rashba, Properties of semiconductors with an extremum loop. 1. Cyclotron and combinational resonance in a magnetic field perpendicular to the plane of the loop, Sov. Phys. Solid State 2, 1109 (1960).

[17] Y. Bychkov and E. Rashba, Properties of a 2D electron gas with lifted spectral degeneracy, JETP Lett. 39, 78 (1984).

[18] Y. A. Bychkov and E. I. Rashba, Oscillatory effects and the magnetic susceptibility of carriers in inversion layers, J. Phys. C: Solid State Phys. 17, 6039 (1984).

[19] V. V. Bryksin and P. Kleinert, Theory of electric-field-induced spin accumulation and spin current in the two-dimensional Rashba model, Phys. Rev. B 73, 165313 (2006).

[20] Y. K. Kato, R. C. Myers, A. C. Gossard, and D. D. Awschalom, Current-Induced Spin Polarization in Strained Semiconductors, Phys. Rev. Lett. 93, 176601 (2004).

[21] A. Y. Silov, P. A. Blajnov, J. H. Wolter, R. Hey, K. H. Ploog, and N. S. Averkiev, Current-induced spin polarization at a single heterojunction, Appl. Phys. Lett. 85, 5929 (2004).

[22] S. Ganichev, S. Danilov, P. Schneider, V. Bel'kov, L. Golub, W. Wegscheider, D. Weiss, and W. Prettl, Electric current-induced spin orientation in quantum well structures, J. Magn. Magn. Mater. 300, 127 (2006). 
[23] P. Kleinert, V. V. Bryksin, and O. Bleibaum, Spin accumulation in lateral semiconductor superlattices induced by a constant electric field, Phys. Rev. B 72, 195311 (2005).

[24] D. V. Khomitsky, Electric-field induced spin textures in a superlattice with Rashba and Dresselhaus spin-orbit coupling, Phys. Rev. B 79, 205401 (2009).

[25] D. Culcer, E. H. Hwang, T. D. Stanescu, and S. Das Sarma, Two-dimensional surface charge transport in topological insulators, Phys. Rev. B 82, 155457 (2010).

[26] A. R. Mellnik, J. S. Lee, A. Richardella, J. L. Grab, P. J. Mintun, M. H. Fischer, A. Vaezi, A. Manchon, E.-A. Kim, N. Samarth, and D. C. Ralph, Spin-transfer torque generated by a topological insulator, Nature (London) 511, 449 (2010).

[27] D. Pesin and A. H. MacDonald, Spintronics and pseudospintronics in graphene and topological insulators, Nat. Mater. 11, 409 (2012).

[28] Y. Ando, T. Hamasaki, T. Kurokawa, K. Ichiba, F. Yang, M. Novak, S. Sasaki, K. Segawa, Y. Ando, and M. Shiraishi, Electrical detection of the spin polarization due to charge flow in the surface state of the topological insulator $\mathrm{Bi}_{1.5} \mathrm{Sb}_{0.5} \mathrm{Te}_{1.7} \mathrm{Se}_{1.3}$, Nano Lett. 14, 6226 (2014).

[29] C. H. Li, O. M. J. van 't Erve, J. T. Robinson, Y. Liu, L. Li, and $\mathrm{B}$. T. Jonker, Electrical detection of charge-current-induced spin polarization due to spin-momentum locking in $\mathrm{Bi}_{2} \mathrm{Se}_{3}$, Nat. Nanotechnol. 9, 218 (2014).

[30] J. Tian, I. Miotkowski, S. Hong, and Y. P. Chen, Electrical injection and detection of spin-polarized currents in topological insulator $\mathrm{Bi}_{2} \mathrm{Te}_{2} \mathrm{Se}$, Sci. Rep. 5, 14293 (2015).

[31] J.-C. Rojas-Sánchez, S. Oyarzún, Y. Fu, A. Marty, C. Vergnaud, S. Gambarelli, L. Vila, M. Jamet, Y. Ohtsubo, A. TalebIbrahimi, P. Le Fèvre, F. Bertran, N. Reyren, J.-M. George, and A. Fert, Spin to Charge Conversion at Room Temperature by Spin Pumping into a New Type of Topological Insulator: $\alpha$-Sn Films, Phys. Rev. Lett. 116, 096602 (2016).

[32] S. Zhang and A. Fert, Conversion between spin and charge currents with topological insulators, Phys. Rev. B 94, 184423 (2016).

[33] M. Rodriguez-Vega, G. Schwiete, J. Sinova, and E. Rossi, Giant Edelstein effect in topological-insulator-graphene heterostructures, Phys. Rev. B 96, 235419 (2017).

[34] A. Johansson, J. Henk, and I. Mertig, Edelstein effect in Weyl semimetals, Phys. Rev. B 97, 085417 (2018).

[35] E. Lesne, Y. Fu, S. Oyarzun, J. C. Rojas-Sánchez, D. C. Vaz, H. Naganuma, G. Sicoli, J.-P. Attané, M. Jamet, E. Jacquet, J.-M. George, A. Barthélémy, H. Jaffrès, A. Fert, M. Bibes, and L. Vila, Highly efficient and tunable spin-to-charge conversion through Rashba coupling at oxide interfaces, Nat. Mater. 15, 1261 (2016).

[36] G. Seibold, S. Caprara, M. Grilli, and R. Raimondi, Theory of the Spin Galvanic Effect at Oxide Interfaces, Phys. Rev. Lett. 119, 256801 (2017).

[37] Q. Song, H. Zhang, T. Su, W. Yuan, Y. Chen, W. Xing, J. Shi, J. Sun, and W. Han, Observation of inverse Edelstein effect in Rashba-split 2DEG between $\mathrm{SrTiO}_{3}$ and $\mathrm{LaAlO}_{3}$ at room temperature, Sci. Adv. 3, e1602312 (2017).

[38] D. C. Vaz, P. Noël, A. Johansson, B. Göbel, F. Y. Bruno, G. Singh, S. McKeown-Walker, F. Trier, L. M. Vicente-Arche, A. Sander, S. Valencia, P. Bruneel, M. Vivek, M. Gabay, N. Bergeal, F. Baumberger, H. Okuno, A. Barthélémy, A. Fert, L. Vila, I. Mertig, J.-P. Attané, and M. Bibes, Mapping spin- charge conversion to the band structure in a topological oxide two-dimensional electron gas, Nat. Mater. 18, 1187 (2019).

[39] M.-C. Chang and Q. Niu, Berry phase, hyperorbits, and the Hofstadter spectrum: Semiclassical dynamics in magnetic Bloch bands, Phys. Rev. B 53, 7010 (1996).

[40] S. Zhang and Z. Yang, Intrinsic Spin and Orbital Angular Momentum Hall Effect, Phys. Rev. Lett. 94, 066602 (2005).

[41] T. Tanaka, H. Kontani, M. Naito, T. Naito, D. S. Hirashima, K. Yamada, and J. Inoue, Intrinsic spin Hall effect and orbital Hall effect in $4 d$ and $5 d$ transition metals, Phys. Rev. B 77, 165117 (2008).

[42] H. Kontani, T. Tanaka, D. S. Hirashima, K. Yamada, and J. Inoue, Giant Intrinsic Spin and Orbital Hall Effects in $\mathrm{Sr}_{2} M \mathrm{O}_{4}$ ( $M=\mathrm{Ru}, \mathrm{Rh}, \mathrm{Mo}$ ), Phys. Rev. Lett. 100, 096601 (2008).

[43] H. Kontani, T. Tanaka, D. S. Hirashima, K. Yamada, and J. Inoue, Giant Orbital Hall Effect in Transition Metals: Origin of Large Spin and Anomalous Hall Effects, Phys. Rev. Lett. 102, 016601 (2009).

[44] J.-P. Hanke, F. Freimuth, S. Blügel, and Y. Mokrousov, Prototypical topological orbital ferromagnet $\gamma$-FeMn, Sci. Rep. 7, 41078 (2017).

[45] D. Jo, D. Go, and H.-W. Lee, Gigantic intrinsic orbital Hall effects in weakly spin-orbit coupled metals, Phys. Rev. B 98, 214405 (2018).

[46] L. S. Levitov, Y. V. Nazarov, and G. M. Éliashberg, Magnetoelectric effects in conductors with mirror isomer symmetry, Sov. Phys. JETP 61, 133 (1985).

[47] T. Yoda, T. Yokoyama, and S. Murakami, Current-induced orbital and spin magnetizations in crystals with helical structure, Sci. Rep. 5, 12024 (2015).

[48] T. Yoda, T. Yokoyama, and S. Murakami, Orbital Edelstein effect as a condensed-matter analog of solenoids, Nano Lett. 18, 916 (2018).

[49] D. Go, J.-P. Hanke, P. M. Buhl, F. Freimuth, G. Bihlmayer, H.-W. Lee, Y. Mokrousov, and S. Blügel, Toward surface orbitronics: Giant orbital magnetism from the orbital Rashba effect at the surface of $s p$-metals, Sci. Rep. 7, 46742 (2017).

[50] L. Salemi, M. Berritta, A. K. Nandy, and P. M. Oppeneer, Orbitally dominated Rashba-Edelstein effect in noncentrosymmetric antiferromagnets, Nat. Commun. 10, 5381 (2019).

[51] A. Ohtomo and H. Y. Hwang, A high-mobility electron gas at the $\mathrm{LaAlO}_{3} / \mathrm{SrTiO}_{3}$ heterointerface, Nature (London) 427, 423 (2004).

[52] S. Thiel, G. Hammerl, A. Schmehl, C. W. Schneider, and J. Mannhart, Tunable quasi-two-dimensional electron gases in oxide heterostructures, Science 313, 1942 (2006).

[53] A. D. Caviglia, S. Gariglio, N. Reyren, D. Jaccard, T. Schneider, M. Gabay, S. Thiel, G. Hammerl, J. Mannhart, and J.-M. Triscone, Electric field control of the $\mathrm{LaAlO}_{3} / \mathrm{SrTiO}_{3}$ interface ground state, Nature (London) 456, 624 (2008).

[54] T. C. Rödel, F. Fortuna, S. Sengupta, E. Frantzeskakis, P. L. Fèvre, F. Bertran, B. Mercey, S. Matzen, G. Agnus, T. Maroutian, P. Lecoeur, and A. F. Santander-Syro, Universal fabrication of 2D electron systems in functional oxides, Adv. Mater. 28, 1976 (2016).

[55] A. B. Posadas, K. J. Kormondy, W. Guo, P. Ponath, J. GelerKremer, T. Hadamek, and A. A. Demkov, Scavenging of oxygen from $\mathrm{SrTiO}_{3}$ during oxide thin film deposition and the formation of interfacial 2DEGs, J. Appl. Phys. 121, 105302 (2017). 
[56] Z. Zhong, A. Tóth, and K. Held, Theory of spin-orbit coupling at $\mathrm{LaAlO}_{3} / \mathrm{SrTiO}_{3}$ interfaces and $\mathrm{SrTiO}_{3}$ surfaces, Phys. Rev. B 87, 161102(R) (2013).

[57] G. Khalsa, B. Lee, and A. H. MacDonald, Theory of $t_{2 g}$ electron-gas Rashba interactions, Phys. Rev. B 88, 041302(R) (2013).

[58] M. Vivek, M. O. Goerbig, and M. Gabay, Topological states at the (001) surface of $\mathrm{SrTiO}_{3}$, Phys. Rev. B 95, 165117 (2017).

[59] C. L. Kane and E. J. Mele, $Z_{2}$ Topological Order and the Quantum Spin Hall Effect, Phys. Rev. Lett. 95, 146802 (2005).

[60] J. Henk, A. Ernst, and P. Bruno, Spin polarization of the $L$-gap surface states on $\mathrm{Au}(111)$, Phys. Rev. B 68, 165416 (2003).

[61] M. Hoesch, M. Muntwiler, V. N. Petrov, M. Hengsberger, L. Patthey, M. Shi, M. Falub, T. Greber, and J. Osterwalder, Spin structure of the Shockley surface state on Au(111), Phys. Rev. B 69, 241401(R) (2004).

[62] J. Henk, M. Hoesch, J. Osterwalder, A. Ernst, and P. Bruno, Spin-orbit coupling in the L-gap surface states of $\mathrm{Au}(111)$ : Spin-resolved photoemission experiments and first-principles calculations, J. Phys.: Condens. Matter 16, 7581 (2004).

[63] Y. K. Kato, R. C. Myers, A. C. Gossard, and D. D. Awschalom, Coherent spin manipulation without magnetic fields in strained semiconductors, Nature (London) 427, 50 (2004).

[64] V. Sih, R. C. Myers, Y. K. Kato, W. H. Lau, A. C. Gossard, and D. D. Awschalom, Spatial imaging of the spin Hall effect and current-induced polarization in two-dimensional electron gases, Nat. Phys. 1, 31 (2005).

[65] M. Johnson, Theory of spin-dependent transport in ferromagnet-semiconductor heterostructures, Phys. Rev. B 58, 9635 (1998).

[66] P. R. Hammar and M. Johnson, Potentiometric measurements of the spin-split subbands in a two-dimensional electron gas, Phys. Rev. B 61, 7207 (2000).

[67] J. C. Rojas Sánchez, L. Vila, G. Desfonds, S. Gambarelli, J. P. Attané, J. M. De Teresa, C. Magén, and A. Fert, Spin-to-charge conversion using Rashba coupling at the interface between nonmagnetic materials, Nat. Commun. 4, 2944 (2013).

[68] Y. Tserkovnyak, A. Brataas, and G. E. W. Bauer, Enhanced Gilbert Damping in Thin Ferromagnetic Films, Phys. Rev. Lett. 88, 117601 (2002).

[69] L. Liu, T. Moriyama, D. C. Ralph, and R. A. Buhrman, SpinTorque Ferromagnetic Resonance Induced by the Spin Hall Effect, Phys. Rev. Lett. 106, 036601 (2011).
[70] K. Kondou, R. Yoshimi, A. Tsukazaki, Y. Fukuma, J. Matsuno, K. S. Takahashi, M. Kawasaki, Y. Tokura, and Y. Otani, Fermilevel-dependent charge-to-spin current conversion by Dirac surface states of topological insulators, Nat. Phys. 12, 1027 (2016).

[71] D. C. Vaz, F. Trier, A. Dyrdał, A. Johansson, K. Garcia, A. Barthélémy, I. Mertig, J. Barnaś, A. Fert, and M. Bibes, Determining the Rashba parameter from the bilinear magnetoresistance response in a two-dimensional electron gas, Phys. Rev. Materials 4, 071001(R) (2020).

[72] J. Stöhr and H. Siegmann, Magnetism: From Fundamentals to Nanoscale Dynamics, Springer Series in Solid-State Sciences (Springer, Berlin, Heidelberg, 2007).

[73] B. T. Thole, P. Carra, F. Sette, and G. van der Laan, X-ray Circular Dichroism as a Probe of Orbital Magnetization, Phys. Rev. Lett. 68, 1943 (1992).

[74] G. van der Laan, Angular momentum sum rules for x-ray absorption, Phys. Rev. B 57, 112 (1998).

[75] S. Manipatruni, D. E. Nikonov, C.-C. Lin, T. A. Gosavi, H. Liu, B. Prasad, Y.-L. Huang, E. Bonturim, R. Ramesh, and I. A. Young, Scalable energy-efficient magnetoelectric spinorbit logic, Nature (London) 565, 35 (2019).

[76] L. Petersen and P. Hedegård, A simple tight-binding model of spin-orbit splitting of $s p$-derived surface states, Surf. Sci. 459, 49 (2000).

[77] M. Ben Shalom, M. Sachs, D. Rakhmilevitch, A. Palevski, and Y. Dagan, Tuning Spin-Orbit Coupling and Superconductivity at the $\mathrm{SrTiO}_{3} / \mathrm{LaAlO}_{3}$ Interface: A Magnetotransport Study, Phys. Rev. Lett. 104, 126802 (2010).

[78] A. Fête, S. Gariglio, C. Berthod, D. Li, D. Stornaiuolo, M. Gabay, and J.-M. Triscone, Large modulation of the Shubnikov-de Haas oscillations by the Rashba interaction at the $\mathrm{LaAlO}_{3} / \mathrm{SrTiO}_{3}$ interface, New J. Phys. 16, 112002 (2014).

[79] C. Cancellieri, A. S. Mishchenko, U. Aschauer, A. Filippetti, C. Faber, O. S. Barišić, V. A. Rogalev, T. Schmitt, N. Nagaosa, and V. N. Strocov, Polaronic metal state at the $\mathrm{LaAlO}_{3} / \mathrm{SrTiO}_{3}$ interface, Nat. Commun. 7, 10386 (2016).

[80] A. Jouan, G. Singh, E. Lesne, D. C. Vaz, M. Bibes, A. Barthélémy, C. Ulysse, D. Stornaiuolo, M. Salluzzo, S. Hurand, J. Lesueur, C. Feuillet-Palma, and N. Bergeal, Quantized conductance in a one-dimensional ballistic oxide nanodevice, Nat. Electron. 3, 201 (2020). 